expose a student or patient to an unacceptable level of risk. We have therefore developed an immersive simulation course that aims to enhance undergraduate psychiatry training.

Method. Our course was developed by medical education faculty and psychiatry staff. The course handbook includes storyboards, patient scripts and debrief guidelines. Clinical scenarios are mapped to university intended learning outcomes and include; conducting a risk assessment for a patient with emotionally unstable personality disorder and comorbid depression, managing a manic patient in the Emergency Department and assessing a patient with obsessive-compulsive disorder who has developed skin damage due to hand washing.

The one-day course is delivered to groups of 4-8 students from the Universities of Glasgow and Edinburgh during their placements in NHS Lanarkshire. The course takes place in a simulation suite and is facilitated by psychiatrists and medical education faculty. Students each take the lead role during a clinical scenario in which they will encounter a simulated patient. Live video from the simulation is broadcast to other candidates. Scenarios last 10-15 minutes with a 20-30 minute group debrief immediately afterwards. The debrief utilises the PEARLS framework (Promoting Excellence and Reflective Learning in Simulation) and provides the opportunity for peer and facilitator feedback, as well as discussions regarding mental state examination, diagnosis and management.

Result. Qualitative and quantitative feedback has been collected via an anonymous electronic post-course questionnaire. To date, the course has received universally positive feedback. 93\% of candidates rated the overall quality of the course as a learning experience as 'excellent'. Students reported that the course helped them develop communication skills which they could apply to future clinical situations. In addition, candidates felt participation had increased their confidence in taking a psychiatric history and performing a risk assessment.

Conclusion. Immersive simulation is an underutilised tool in psychiatry education. Our course complements the existing educational programme of lectures and ward-based teaching and has been positively received. It provides the opportunity for students to develop interview techniques and communication skills in a safe, controlled environment.

\section{Bedside teaching: an invaluable tool in undergraduate medical education}

\section{Kenneth Ruddock* \\ NHS Lanarkshire \\ ${ }^{*}$ Corresponding author.}

\section{doi: 10.1192/bjo.2021.431}

Aims. Bedside teaching is one of the most important modalities in medical education. Sir William Osler stated, "Medicine is learned by the bedside and not in the classroom". Despite this, the use of bedside teaching in the undergraduate curriculum has been declining, potentially due to changes in course design, increasing clinical workloads and reducing inpatient numbers. In my role as a Clinical Teaching Fellow (CTF), I have aimed to maximise bedside teaching and promote it as the primary approach for student learning.

Method. As a CTF, I deliver teaching to students from the Universities of Glasgow and Edinburgh during their placements in NHS Lanarkshire. Weekly teaching is provided to groups of 2-4 students, with around $50 \%$ of sessions delivered 'at the bedside'.

Within psychiatry, there is a vast range of potential bedside teaching topics. Given the length of time required to conduct a full psychiatric history and mental state examination (MSE), teaching sessions instead focus on one specific component of the patient interview, for example, assessing perceptual abnormalities or delusions, conducting a substance use history or exploring social circumstances and the functional impact of illness. This approach allows for more focussed feedback and teaching. Session structure is based upon Cox's model of bedside teaching, which I have modified slightly for the psychiatry setting.

Student feedback has been collected via an anonymous electronic end-of-block questionnaire.

Result. Qualitative feedback reveals that students in NHS Lanarkshire value bedside teaching, with one student describing it as "informative, comprehensive and relevant for upcoming exams and clinical practice".

There are a number of potential barriers to consider when delivering bedside teaching in psychiatry. These include issues identifying suitable patients who can provide informed consent to participate and the ethical concerns regarding exploring difficult subjects such as suicide risk assessment with patients for purely educational purposes.

These issues can be overcome; in inpatient units, there is usually a small cohort of patients who are able to consent and engage in student teaching, and difficult subjects can alternatively be addressed during role-play or simulation sessions.

Conclusion. Despite its challenges, bedside teaching can be an enjoyable and rewarding approach in undergraduate medical education, with feedback revealing it is positively received in NHS Lanarkshire. By utilising Cox's model and focussing on specific aspects of MSE and history-taking, bedside teaching is more accessible and an invaluable tool for psychiatric teaching. Clinicians and educators are encouraged to keep the patient at the centre of student learning.

\section{Balint in the time of COVID-19: participant and facilitator experience of virtual Balint groups compared with in-person}

\section{Sheliza Samnani* and Masud Awal \\ Birmingham and Solihull Mental Health Foundation Trust ${ }^{*}$ Corresponding author.}

doi: 10.1192/bjo.2021.432

Aims. Our Trust increased Balint group provision, relocating virtually for psychiatry doctors to explore the emotional impact of clinical practice and doctor-patient relationships, during unfamiliar challenges of the pandemic. This unique context allowed comparison of multiple virtual and face-to-face (F2F) Balint-type group experiences for participants and facilitators.

Method. In March 2020, existing core trainee (CT) year 1 and 2, higher trainee (ST) and consultant Balint groups became virtual, with new CT3 and Speciality Doctor and Associate Specialist (SAS) virtual Balint groups established.

All 57 participants and 5 facilitators were sent an anonymous electronic survey to retrospectively rate virtual Balint (March-August 2020) and their preceding F2F Balint group (suggesting September 2019-February 2020) experience.

Result. The response rate was $89 \%$ for participants (51 respondents) and $100 \%$ for facilitators (5 respondents).

For group participants, 90\% (virtual) and 78\% (F2F) agreed or strongly agreed that Balint group provided an opportunity to explore challenging aspects of clinical work. $76 \%$ (virtual) and $71 \%$ (F2F) agreed or strongly agreed that it made them feel more supported. Almost 50\% agreed or strongly agreed that virtual and F2F Balint group helped work feel less stressful. Both 